\title{
NOUVELLe
}

\section{Un passager clandestin}

\section{Un VIH infectieux caché dans les plaquettes alimente les macrophages tissulaires}

Fernando Real ${ }^{1-3}$, Aiwei Zhu ${ }^{1-3}$, Morgane Bomsel ${ }^{1-3}$

\author{
${ }^{1}$ Laboratoire Entrée muqueuse du VIH et immunité muqueuse, \\ Département 3I, Institut Cochin, Université Paris Descartes, \\ Sorbonne Paris Cité, 75014 Paris, France. \\ ${ }^{2}$ CNRS UMR8104, 75014 Paris, France. \\ ${ }^{3}$ Inserm U1016, Institut Cochin, 22 rue Méchain, 75014 Paris, \\ France. \\ morgane.bomsel@inserm.fr
}

$>$ Chez les patients infectés par le virus de l'immunodéficience humaine (VIH) sous traitement antiviral efficace, avec une virémie indétectable dans le sang, le virus arrête sa réplication mais reste intégré dans l'ADN de cellules appelées « réservoirs du virus ». Plusieurs types cellulaires peuvent former des réservoirs, les mieux connus étant les lymphocytes T CD4 ${ }^{+}$circulants [1]. Cependant les macrophages tissulaires sont aussi capables de constituer des réservoirs du VIH, comme nous l'avons montré récemment [2]. Les mécanismes qui permettent aux réservoirs du virus de se maintenir au cours du temps (jusqu'à plusieurs dizaines d'années) malgré les traitements antirétroviraux restent mal compris. En plus de leur implication dans l'hémostase, les plaquettes sanguines ont un rôle dans la réponse immunitaire dont l'importance commence à être reconnue. En particulier, les plaquettes sont capables d'internaliser des agents infectieux bactériens ou viraux, comme le VIH ou les virus de l'herpès, in vitro [3]. Si tel était le cas in vivo, ces virus seraient alors abrités par la plaquette, protégés des agressions extérieures, tout en circulant dans l'organisme et pouvant atteindre les tissus. Par ailleurs, les plaquettes sont éliminées par les macrophages tissulaires dans des contextes aussi bien physiologiques qu'inflammatoires $[4,5]$, et la phagocytose des plaquettes contenant du virus par les macrophages représenterait donc une voie permettant au VIH d'établir et de maintenir des réservoirs viraux dans ces cellules $[2,6](\rightarrow)$

$(\rightarrow)$ Voir la Nouvelle de F. Real et M. Bomsel, $m / s n^{\circ} 3$, mars 2019 , page 209
L'étude dont nous avons récemment publié les résultats dans la revue Science Translational Medicine montre que les plaquettes sanguines de patients infectés par le VIH hébergent des virus infectieux, malgré une thérapie antivirale combinée efficace supprimant la charge virale dans le sang. Chez ces patients, la présence de VIH dans les plaquettes est fortement corrélée à l'échec du rétablissement de la réponse immunitaire impliquant les lymphocytes T $\mathrm{CD}^{+}$. De plus, les plaquettes peuvent propager le virus infectieux aux macrophages in vitro, ce qui révèle l'existence d'une voie alternative de dissémination du VIH vers ce réservoir cellulaire du virus [7].

In vivo, les plaquettes sanguines de sujets infectés par le VIH sous traitement antirétroviral, efficace contiennent du virus infectieux Nous avons d'abord testé l'hypothèse selon laquelle les plaquettes se comportent en vecteur circulant du VIH in vivo, mettant le virus à l'abri de l'action directe des médicaments antirétroviraux, ce qui favoriserait sa propagation dans l'organisme. Ainsi, nous avons analysé du plasma riche en plaquettes provenant de personnes infectées par le VIH et recevant un traitement antirétroviral efficace. Nous nous sommes concentrés sur des patients ayant une charge virale dans le sang indétectable pendant plus d'un an, à la date du prélèvement de plaquettes, que nous appellerons patients «virologiquement supprimés». À l'aide de ces prélèvements de plaquettes, nous avons mon- tré par une combinaison de techniques - PCR quantitative, cytométrie en flux couplée l'hybridation in situ, immunomarquages détectés par microscopies confocale et électronique - que les plaquettes de ces patients contiennent du VIH, non seulement de I'ARN viral mais aussi des protéines comme la protéine de capside p24, le virus étant toujours localisé dans des vésicules internes. Nous avons ensuite montré que ce virus est infectieux et capable de transférer l'infection à des macrophages tissulaires in vitro [7] (Figure 1). L'infection de ces macrophages est productive car les macrophages tissulaires secrètent à leur tour des virus infectieux. Comment alors empêcher la transmission par les plaquettes de cette infection aux macrophages tissulaires? En bloquant l'interaction entre les plaquettes et les macrophages avec des agents antiagrégants plaquettaires tels que les anticorps monoclonaux (fragment Fab) spécifiques des intégrines des plaquettes, comme l'intégrine $\alpha \mathrm{llb} / \beta 3$ (abciximab) [7].

La présence du VIH dans les plaquettes est fortement corrélée à une défaillance immunologique

Environ $20 \%$ des patients sous traitement antirétroviral efficace et «virologiquement supprimés » ne parviennent pas à reconstituer un taux de lymphocytes T CD4 $4^{+}$suffisant pour leur conférer un statut de compétence immunitaire. Ces patients, appelés «non-répondeurs immunologiques » (NRI) [8], sont définis comme des individus infectés par 
A

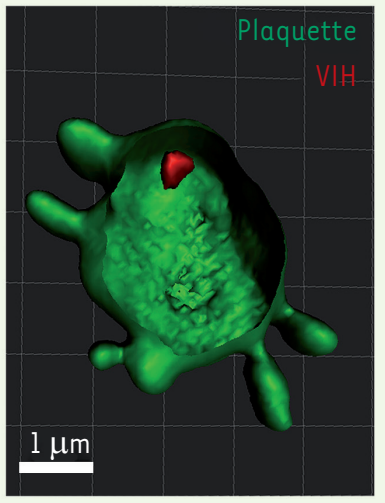

B

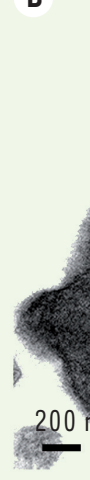

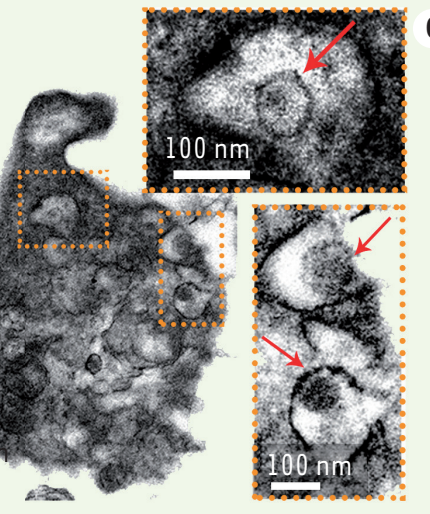

12
D
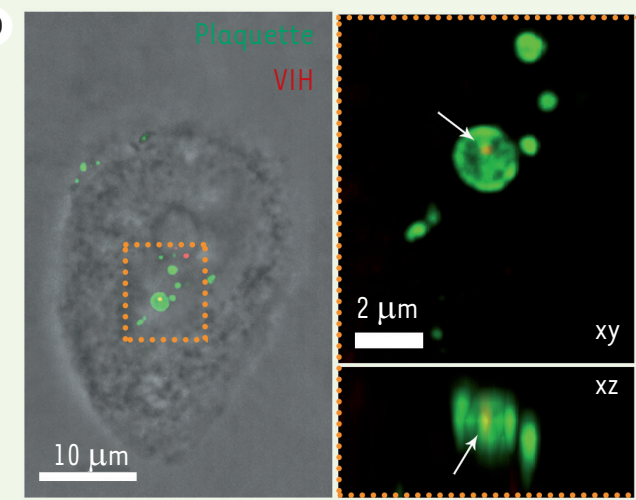

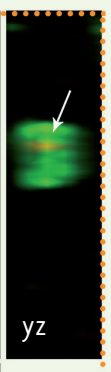

$y z$

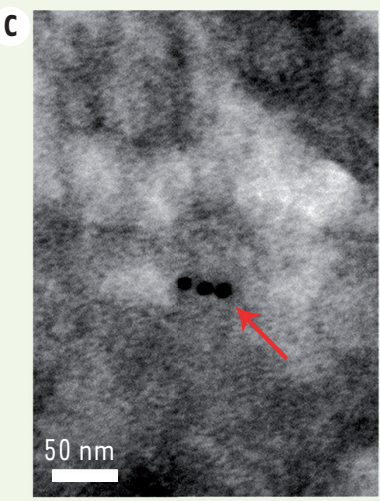

$\varepsilon$

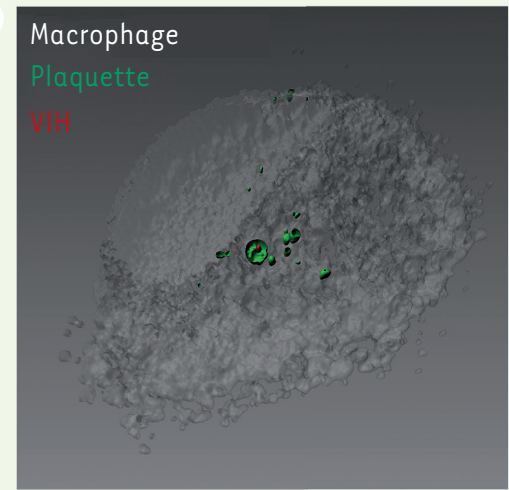

$\mathbf{F}$

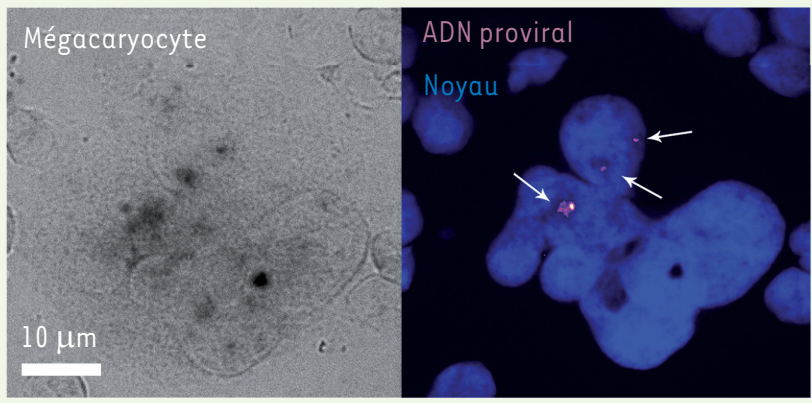

G

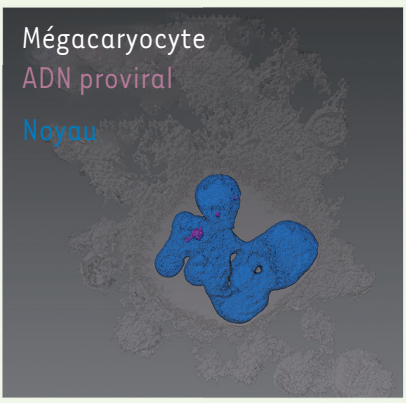

Figure 1. Les plaquettes d'individus infectés par le VIH, sous traitement antirétroviral efficace, contiennent du VIH infectieux. A. Image d'une plaquette contenant du virus détecté par immunomarquage (observation par microscopie confocale à fort grossissement, et reconstruction tridimensionnelle de l'image). Les plaquettes sont caractérisées par l'expression de CD4l (en vert) et le VIH par celle de la protéine p24 (en rouge). B. Image, par microscopie électronique à transmission, d'une plaquette, congelée puis décongelée, contenant du VIH. Les particules virales sont des structures de forme ronde de diamètre 70 à $120 \mathrm{~nm}$, avec un centre dense aux électrons de diamètre 20 à $60 \mathrm{~nm}$. Trois de ces particules sont visibles à l'intérieur des deux cadres en pointillé orange, et sont indiquées par une flèche rouge dans des images agrandies de ces cadres. $C$. Image d'un virus contenu dans un compartiment (voir commentaires dans le texte) d'une plaquette observée par microscopie électronique après cryocongélation et immunomarquage. Le virus, d'un diamètre d'environ $100 \mathrm{~nm}$ (flèche rouge), est identifié par immunomarquage avec un anticorps anti-p24 couplé à l'or colloïdal (points noirs). D. Image, par microscopie confocale en contraste de phase, d'un macrophage après endocytose d'une plaquette (identifiée par immunomarquage avec un anticorps dirigé contre CD41, en vert) contenant le VIH (identifié par immunomarquage avec un anticorps dirigé contre p24, en rouge). Le cadre orange en pointillé indique la zone qui est agrandie dans l'encart (à droite) montrant des projections tridimensionnelles (xy, xz et yz) de la plaquette contenant le VIH. La flèche blanche indique le virus immunomarqué. $\varepsilon$. Reconstruction tridimensionnelle de l'image confocale montrée en D. F. Image en contraste de phase (à gauche) et image fusionnée (deux canaux de fluorescence) (à droite) d'un mégacaryocyte dans lequel le génome du VIH (coloré en magenta et indiqué par des flèches blanches) s'est intégré à l'ADN cellulaire contenu dans le noyau polylobé (coloré en bleu par DAPI). G. Reconstruction tridimensionnelle de l'image confocale montrée en $F$. 
le VIH et dont le nombre de lymphocytes $\mathrm{T}^{\mathrm{CD}} 4^{+}$dans le sang est inférieur à 350 cellules/ $\mu$ l malgré une charge virale sanguine indétectable depuis plus d'un an [9]. Les causes de cette défaillance immunologique restent inconnues, et aucun traitement n'est disponible pour améliorer la restauration du nombre de lymphocytes $\mathrm{T} \mathrm{CD4}^{+}$et la santé de ces patients NRI. À cause de leur faible taux de lymphocytes T CD4 $4^{+}$, les patients NRI sont plus exposés aux risques de morbidité et de mortalité liées au Sida (syndrome d'immunodéficience acquise) et à d'autres maladies.

Nous avons analysé les données cliniques de l'ensemble des patients durant 18 mois avant et 18 mois après avoir recueilli et analysé la présence du virus dans leurs plaquettes. Ces analyses ont montré que la présence du VIH infectieux dans les plaquettes est indépendante de la virémie du patient et du nombre de ses plaquettes. En revanche, elle est fortement corrélée avec la valeur la plus basse de la concentration des lymphocytes $T$ $\mathrm{CD}^{+}$dans le sang atteinte pendant les 36 mois de suivi du patient ( $<200$ lymphocytes $\left.\mathrm{T} \mathrm{CD}^{+} / \mu \mathrm{l}\right)$ et la faible concentration de ces lymphocytes à la date du prélèvement ( 350 lymphocytes $\mathrm{T} \mathrm{CD4}^{+} /$ $\mu \mathrm{l})$ [7]. L'analyse multivariée en composantes principales et la régression logistique multinomiale des données montrent que le seul paramètre clinique fortement associé à la présence du VIH dans les plaquettes est un nombre faible de lymphocytes $\mathrm{T} \mathrm{CD}^{+}$. De plus, la présence du VIH dans les plaquettes permet de prédire une absence de remontée du taux de lymphocytes $T$ CD4 ${ }^{+}$chez ces patients pendant les 18 mois suivant l'échantillonnage, ces patients restant NRI. Plus précisément, les analyses statistiques prédictives indiquent que les patients dont les plaquettes sont porteuses du VIH ont une probabilité environ 50 fois plus élevée de ne pas se rétablir immunologiquement, gardant des taux de lymphocytes $T$ inférieurs à $350 / \mu l$ dans les 18 mois qui suivent l'échantillonage malgré la thérapie antirétrovirale, par rapport aux patients dont les plaquettes ne contiennent pas de VIH [7] (Figure 2).

\section{Les mégacaryocytes infectés sont}

la source probable des plaquettes contenant le virus

Comme les patients dont les plaquettes contiennent le VIH n'ont pas de virus détectable dans le sang, cela exclut la possibilité que les plaquettes aient capturé des particules virales provenant du sang par endocytose, bien que l'endocytose des virus ait été montrée in vitro $[10,11]$. En outre, la présence de virus infectieux intacts dans les plaquettes provenant de personnes chez lesquelles la virémie a été supprimée par traitement antirétroviral indique que le VIH s'associe aux plaquettes dans une niche de réplication virale active et que le virus présent dans les plaquettes ne provient pas d'une endocytose de particules virales produites par réplication «à bas bruit » à partir des réservoirs lymphocytaires circulants. Un séquençage du génome du VIH suivi d'analyses phylogénétiques a été effectué à partir d'échantillons longitudinaux de cellules mononucléées du sang et de plaquettes avant le traitement et après une suppression prolongée de la virémie par des antirétroviraux. Cela nous a permis de montrer que les virus contenus dans les plaquettes proviennent d'un site de réplication active, continue, dans un compartiment différent du sang, chez les patients ayant subi une suppression des antirétroviraux.

Quel pouvait être ce compartiment? Les mégacaryocytes (cellules précurseurs des plaquettes), dont l'infection par le VIH avait été suggérée dès la fin des années 1980 par des études d'hybridation in situ détectant I'ARN viral chez des patients infectés et non traités, semblaient le meilleur candidat [12], d'autant plus que leur infection par d'autres types de virus avait également été documentée [13]. Des études récentes ont montré que les précurseurs des mégacaryocytes de la moelle osseuse provenant de patients ayant longtemps été sous traitement antirétroviral contiennent de I'ADN viral capable de se répliquer [14]. Ainsi, contrairement aux plaquettes qui ne peuvent que transporter le VIH et son ARN, les mégacaryocytes infectés dans la moelle osseuse, ayant intégré I'ADN proviral dans leur noyau, pourraient représenter ce site de réplication active du VIH conduisant à l'incorporation de virions ou de I'ARN viral dans les plaquettes lors de leur production (thrombopoïèse). Nous avons alors analysé des prélèvements de moelle osseuse de sujets infectés par le VIH sous traitement antirétroviral efficace, sans virémie détectable, et avec un faible taux de lymphocytes T CD4 ${ }^{+}$. Dans ces échantillons, nous avons détecté I'ARN du VIH et I'ADN proviral intégré dans le noyau des mégacaryocytes par hybridation in situ sur des frottis de moelle osseuse et par une technique de PCR quantitative ( $q P C R$ ) sur des mégacaryocytes provenant de ces patients [7]. Ces résultats indiquent que les mégacaryocytes infectés sont la source la plus probable du virus infectieux présent dans les plaquettes, et qu'ils constituent un réservoir important supplémentaire du VIH, qu'une thérapie antirétrovirale devra aussi éliminer pour être totalement efficace.

\section{Perspectives}

Aucun traitement n'est actuellement disponible pour améliorer la santé des patients non-répondeurs immunologiques, qui sont plus exposés à des risques de morbidité et de mortalité liées au Sida et à d'autres maladies. La forte corrélation entre la présence de plaquettes sanguines contenant le VIH et une mauvaise récupération immunologique sous traitement antirétroviral incite désormais à considérer la détection du VIH dans les plaquettes comme un marqueur prédictif potentiel en pratique médicale. En outre, les résultats de notre étude suggèrent que les inhibiteurs de la fonction pla- 


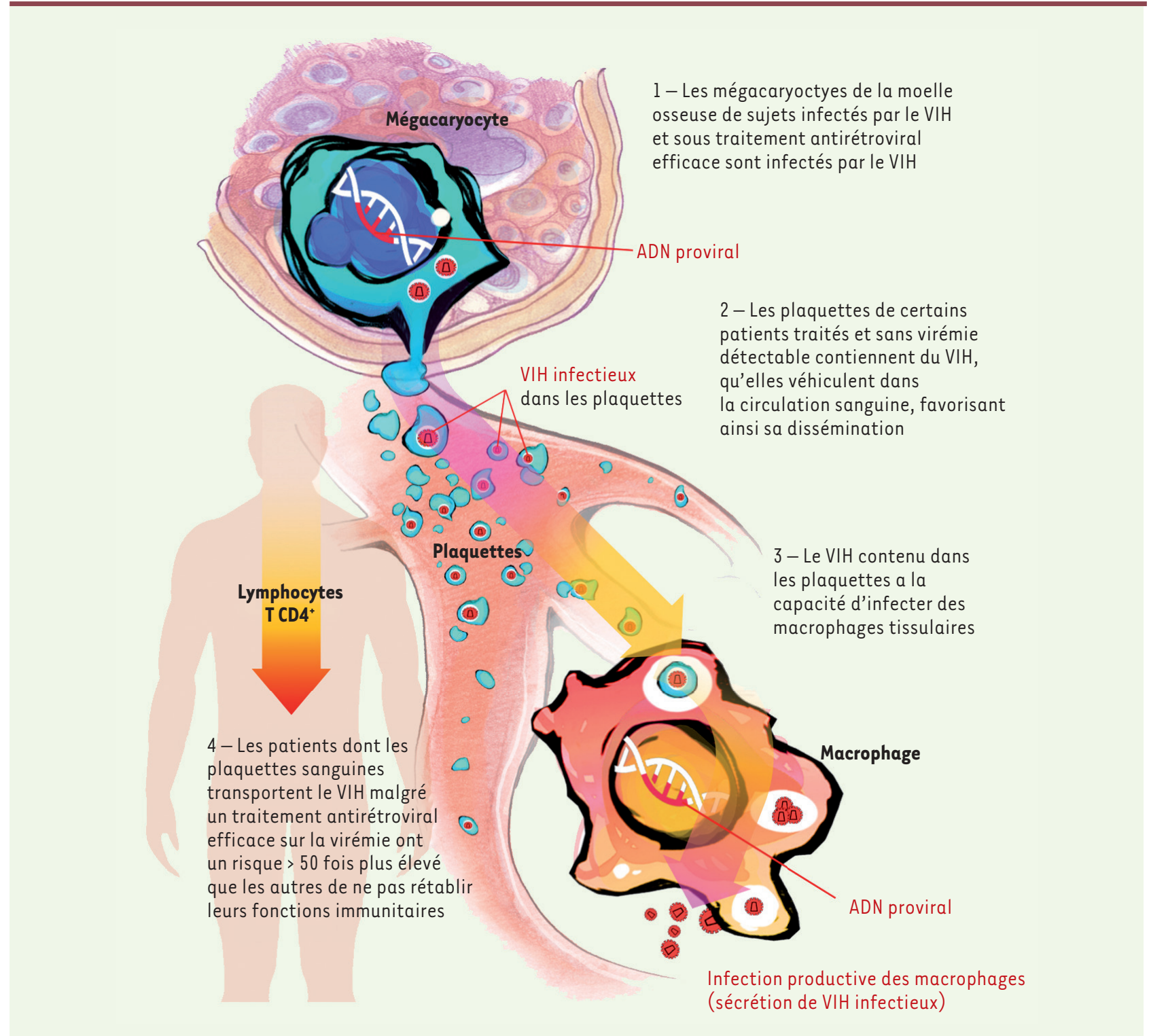

Figure 2. Implication, chez des patients infectés par le VIH, de la présence du virus dans les plaquettes sanguines, dans l'absence de rétablissement immunologique malgré un traitement antirétroviral efficace.

quettaire, largement utilisés dans la prévention des accidents thrombotiques en clinique, pourraient également améliorer l'efficacité de la thérapie antirétrovirale sur la récupération immunologique des patients infectés par le VIH. $\diamond$

A free rider: Infectious HIV hidden in the platelets of infected but virally suppressed patients fuels tissue reservoirs

\section{REMERCIEMENTS}

Ce travail a été soutenu financièrement par l'Agence nationale de recherches sur le SIDA et les hépatites virales (ANRS \# A02015-2-17046) et SIDACTION (\# 15CONV03). FR a bénéficié d'une bourse postdoctorale de la SIDACTION et de l'ANRS, et AZ d'une bourse de doctorat du Chinese Science Council.

\section{LIENS D'INTÉRÊT}

Les auteurs déclarent n'avoir aucun lien d'intérêt concernant les données publiées dans cet article.

\section{RÉFÉRENCES}

1. Chomont N, El-Far M, Ancuta P, et al. HIV reservoir size and persistence are driven by $T$ cell survival and homeostatic proliferation. Nat Med 2009; 15 : 893-900.

2. Ganor Y, Real F, Sennepin A, et al. HIV-1 reservoirs in urethral macrophages of patients unde suppressive antiretroviral therapy. Nat Microbiol $2019 ; 4: 633-44$

3. Garraud 0, Cognasse F. Are platelets cells? And if yes, are they immune cells? Front Immunol $2015 ; 6: 70$.

4. Ballem PJ, Belzberg A, Devine DV, et al. Kinetic studies of the mechanism of thrombocytopenia in patients with human immunodeficiency virus infection. $N$ Engl Med $1992 ; 327: 1779-84$

5. Grozovsky R, Hoffmeister KM, Falet H. Novel clearance mechanisms of platelets. Curr Opin Hematol 2010; 17 : 585-9.

6. Real F, Bomsel M. Visualisation en temps réel de I'infection de la muqueuse génitale par le VIH. Med Sci (Paris) 2019 ; $35: 209-12$

7. Real F, Capron C, Sennepin A, et al. Platelets from HIV-infected individuals on antiretroviral drug therapy with poor $\mathrm{CD}^{+} \mathrm{T}$ cell recovery can harbor replication-competent HIV despite viral suppression. Sci Transl Med 2020 ; 12 : eaat6263 


\section{RÉFÉRENCES}

8. Kaufmann GR, Perrin L, Pantaleo G, et al. CD4 $T$-lymphocyte recovery in individuals with advanced HIV-l infection receiving potent antiretroviral therapy for 4 years: the Swiss HIV cohort study. Arch Intern Med 2003; 163: 2187-95.

9. Ramirez CM, Sinclair $\varepsilon$, Epling L, et al. Immunologic profiles distinguish aviremic HIV-infected adults. AIDS 2016; 30 : 1553-62
10. Chaipan C, Soilleux EJ, Simpson P, et al. DC-SIGN and CLEC-2 mediate human immunodeficiency virus type 1 capture by platelets. J Virol 2006 ; $80: 8951-60$.

11. Youssefian T, Drouin A, Masse JM, et al. Host defense role of platelets: engulfment of HIV and Staphylococcus aureus occurs in a specific subcellular compartment and is enhanced by platelet activation. Blood 2002 ; 99 : 4021-9.

12. Zucker-Franklin D, Cao YZ. Megakaryocytes of human immunodeficiency virus-infected individuals express viral RNA. Proc Natl Acad Sci USA 1989 ; $86: 5595-9$.
13. Vogt MB, Lahon A, Arya RP, et al. Dengue viruses infect human megakaryocytes, with probable clinical consequences. PLoS Negl Trop Dis 2019 ; 13 : e0007837.

14. Sebastian NT, Zaikos TD, Terry V, et al. CD4 is expressed on a heterogeneous subset of hematopoietic progenitors, which persistently harbor CXCR4 and CCR5-tropic HIV proviral genomes in vivo. PLoS Pathog 2017 ; 13 : el006509. 